\title{
Scattering Studies of the Structure of Colloid-Polymer Suspensions and Gels
}

\section{he key step in assembling colloidal particles into useful structures for} applications as coatings, viscosity modifiers, and gels, for example, lies in fine-tuning particle interactions to produce the desired microstructure.

The distinct advantage of adding non-adsorbing polymer to a colloidal suspension (particles of radius $R$ ) lies in the ability to control the microstructure of the resulting dispersion with two independent variables - the molecular weight of the added polymer (radius of gyration $R_{\mathrm{g}}$ ) which controls the range of particle interactions and the concentration of added polymer $\left(c_{\mathrm{p}}\right)$ which controls the strength of particle interactions. Recent studies $[1,2]$ have shown that a wide range of phase behavior can be observed in colloidal dispersions depending on the values of $R_{\mathrm{g}} / R$ and $c_{\mathrm{p}} / c_{\mathrm{p}}{ }^{*}\left(c_{\mathrm{p}}{ }^{*}\right.$ is the polymer overlap concentration). For $R_{\mathrm{g}} / R<0.1$, the suspensions gel as more polymer is added to the system. For $0.1<R_{\mathrm{g}} / R<0.3$, the colloids crystallize and for higher size ratios $\left(R_{\mathrm{g}} / R>0.3\right)$ a liquidliquid phase separation is thermodynamically favored. Standard theories in literature fail to predict the observed trends in phase behavior and we have recently shown that the only model that can qualitatively predict the observed experiments is the polymer reference interaction site model (PRISM) integral equation theory for colloid-polymer mixtures $[1,2]$.

In this work we report on Small Angle Neutron Scattering (SANS) and Ultra Small Angle X-Ray Scattering (USAXS) studies of the structures of model colloidpolymer suspensions over a wide range of $R_{\mathrm{g}} / R$ and $c_{\mathrm{p}} / c_{\mathrm{p}}{ }^{*}$. Our aim in measuring the structure is twofold: to provide a test of different models especially PRISM for predicting the thermodynamics and structure of colloid-polymer mixtures; and to relate the observed microstructure to macroscopic flow properties (shear modulus, viscosity) especially under conditions where the suspensions gel.

The system used in this work consists of $100 \mathrm{~nm}$ diameter silica particles coated with octadecanol (1 $\mathrm{nm}$ to $2 \mathrm{~nm}$ hairs) and suspended in decalin. The particles in the absence of added polymer behave as hard spheres (no interactions except volume exclusion). The polymer used is polystyrene of different molecular weights to provide the appropriate $R_{\mathrm{g}} / R$. Figures 1 and 2 are plots of the mea- sured intensities and the calculated structure factors at an $R_{\mathrm{g}} / R$ of 0.06 and different values of $c_{\mathrm{p}} / c_{\mathrm{p}}{ }^{*}$. The solid lines are comparisons with structure factors from PRISM theory for colloid-polymer mixtures. Agreement with theory and experiment is excellent for samples in the single- phase fluid region thus establishing the fact that PRISM accurately predicts the thermodynamics and structure of these suspensions in the $q$ region of interest.

At a colloid volume fraction of 0.4 and $R_{\mathrm{g}} / R$ of 0.06 , the suspensions gel at a $c_{\mathrm{p}} / c_{\mathrm{p}}{ }^{*}$ of 0.095 . Shown in Fig. 2 is the measured structure factor in the gel phase at a $c_{\mathrm{p}} / c_{\mathrm{p}}{ }^{*}$ of 0.1 . When particles become frozen in the gel state, they fall out of equilibrium and comparisons with theory worsen as is evident from Fig. 2. Another interesting characteristic about these gels is the high upturns in intensity at low angles. Fractal analysis gives an unphysical dimension of 3.4 that led us to propose a model of clusters of particles

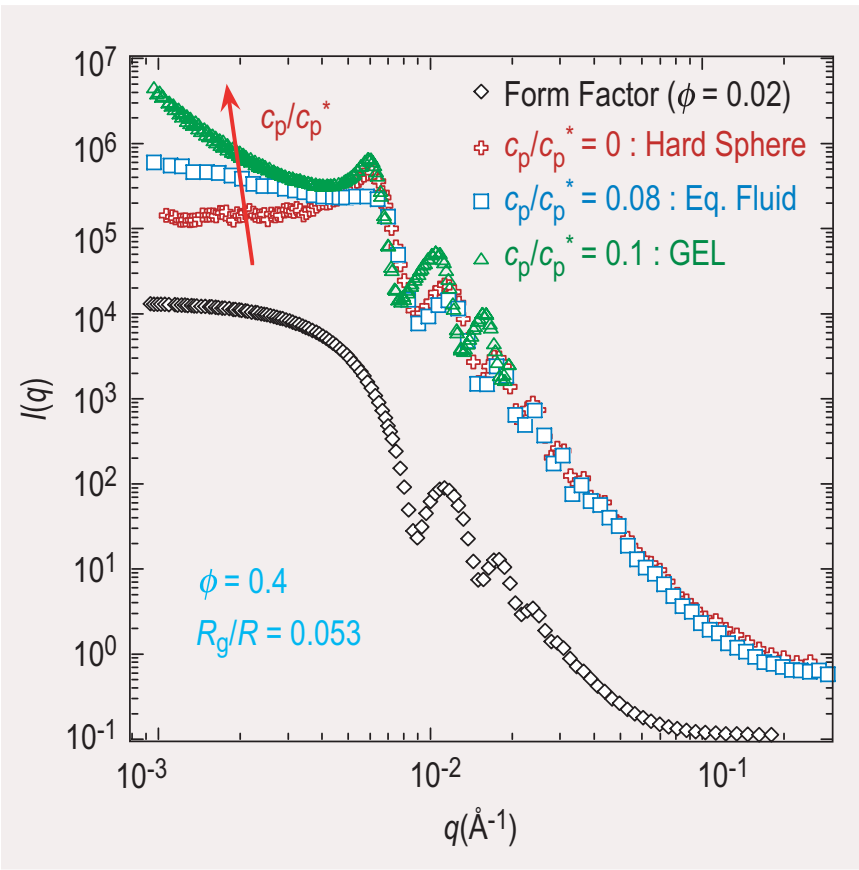

Fig. 1. Normalized scattering intensities $I(q)$ as a function of $q\left(\AA^{-1}\right)$ for samples with varying amounts of polymer quantified by the dimensionless polymer concentration $c_{p} / c_{p}{ }^{*}$. The colloid volume fraction is fixed at $\phi=0.40 \pm 0.01$, and $R_{\mathrm{g}} / R=0.053$. Data is shown for: $c_{p} / c_{p}{ }^{*}=0$ (hard spheres, crosses), 0.08 (equilibrium fluid, squares), and 0.1 (Gel, triangles). Experimental data (diamonds) for the form factor (single particle scattering) is also shown. As more polymer is added to the system, intensities at low angles increase indicating the presence of clusters/aggregates and an increased compressibility. 


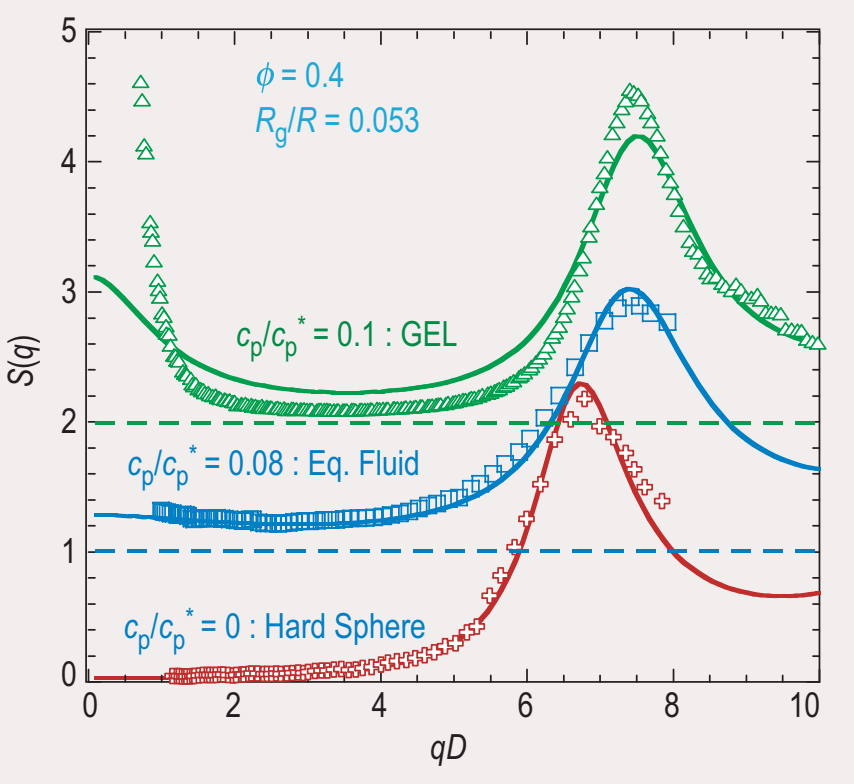

Fig. 2. Structure factor $S(q)$ as a function of the dimensionless wavevector $q D$ (diameter $D=100 \mathrm{~nm}$ ) for samples: $c_{p} / c_{p}{ }^{*}=0.0$ (hard spheres, crosses), 0.08 (equilibrium fluid, squares), and 0.10 (Gel, triangles), for the same $\phi$ and $R_{\mathrm{g}} / R$ as Fig. 1 . Each subsequent data set is offset by 1 for clarity. Dotted lines show relevant baselines signifying $S(q)=0$ for each data set. Solid lines are the zero-adjustable parameter PRISM-mPY theory predictions. The structure factor which depicts the packing of particles with respect to each other in solution is calculated from the data of Fig. 1 by dividing the intensities of the respective suspensions by that of the form factor and then multiplying by the ratio of the volume fractions $\left(\phi_{\text {FormFactor }} / \phi_{\text {Sample }}=0.02 / 0.4\right)[3,4]$. The location of the first peak $\left(q^{*}\right)$ in the structure factor shifts to higher $q^{\prime} s$ as more polymer is added to the system indicating that the particles are getting closer to each other on an average. When the suspension gels, the particles are frozen in space and $q^{*}$ is constant with added polymer as one traverses deeper into the gel $[3,4]$. The increased $S(0)$ for the gel sample is indicative of increased compressibility in the system due to clustering of particles (cluster size $\approx 5-8$ particle diameters). The structure of the gels based on the calculated $S(q)$ is hypothesized to consist of clusters of particles with randomly distributes voids which gives rise to the steep upturns in $S(q)$ at low $q[3,4]$. with randomly distributed voids throughout the gel sample (Debye-Bueche analysis). The cluster size in these samples is $\approx 5$ to 8 particle diameters as calculated from the scattering curves. This finding has an important consequence when trying to predict the flow properties of these suspensions especially the elastic modulus as clusters reduce the ability to store energy compared to an open network of particles. More detailed discussions about the structure and its links to rheology can be found in references [3-5].

The above scattering experiments performed at the NCNR and at Argonne National Laboratory were the first systematic studies of the structure of a model colloidpolymer suspension. These experiments helped us validate the use of PRISM theory for colloid-polymer mixtures. We now have a predictive capability for the variety of interesting phenomena, such as phase transitions between qualitatively different glassy states and reentrant melting of colloidal crystals, observed in these colloid-polymer mixtures.

\section{References}

[1] S. Ramakrishnan, M. Fuchs, K. S. Schweizer and C. F. Zukoski, J. Chem. Phys. 106, 2201 (2002).

[2] S. A. Shah, Y. L. Chen, K. S. Schweizer and C. F. Zukoski, J. Chem. Phys. 118, 3350 (2003).

[3] S. A. Shah, S. Ramakrishnan, Y. L. Chen, K. S. Schweizer and C. F. Zukoski, Langmuir 19(12), 5128 (2003).

[4] S. A. Shah, Y. L. Chen, S. Ramakrishnan, K. S. Schweizer and C. F. Zukoski, J. Phys. Condensed Matter 15(27), 4751 (2003).

[5] S. A. Shah, Y. L. Chen, K. S. Schweizer and C. F. Zukoski, to be published in J. Chem. Phys.
S. Ramakrishnan, S. A. Shah, Y. L. Chen, K. S. Schweizer, and C. F. Zukoski University of Illinois at Urbana-Champaign Urbana, IL 61801 